\title{
TWO NEW SPECIES OF SCAPHYGLOTTIS (ORCHIDACEAE, EPIDENDROIDEAE) FROM COLOMBIA
}

\author{
Dariusz L. SzlachetKo \& Marta Kolanowska ${ }^{1}$
}

\begin{abstract}
Two new species of Scaphyglottis Poepp. \& Endl. from Colombia are described, illustrated and placed within a key for determination of Colombian Scaphyglottis species. The taxonomic affinities of each species are briefly discussed and information about their distribution and ecology is given.
\end{abstract}

Key words: Colombia, orchids, new species, Scaphyglottis, taxonomy

Dariusz L. Szlachetko \& Marta Kolanowska, Department of Plant Taxonomy and Nature Conservation, University of Gdańsk, Wita Stwosza 59,80-308 Gdańsk, Poland; e-mail: martakolanowska@wp.pl

\section{INTRODUCTION}

Since the description of Scaphyglottis Poepp. \& Endl. (Poeppig \& Endlicher 1836) its infrageneric classification has been discussed by taxonomists. Leaf blade and internode shape, lip form, fusion of the lip with the gynostemium, and number of pollinia have been the principle characters considered as bases for delimiting genera - Costaricaea Schltr., Hexisea Lindl., Platyglottis L. O. Williams, Reichenbachanthus Barb. Rodr. and Tetragamestus Rchb. f. Scientists have accepted those taxa in various combinations. Ames (e.g., Ames et al. 1934) recognized Reichenbachanthus and monotypic Costaricaea as synonyms of Hexisea (Dressler 1994), while Adams (1988) limited the latter genus to H. bidentata and H. imbricata. Recent molecular work on the classification of Scaphyglottis (Dressler et al. 2004) confirmed the earlier proposal to conserve the genus in its broad sense (Dressler 1994).

The general characteristics of Scaphyglottis consist in the following: the presence of pseudobulbs arising from the rhizome or apices of older pseudobulbs; resupinate or nonresupinate flowers with free, similar sepals which commonly are wider than the petals; and the usually prominent column-foot.

\footnotetext{
1 Corresponding author
}

Roughly 60 of the $c a 150$ specific names published under Scaphyglottis are accepted presently, and novelties within the genus are still being described (Dressler 2002, 2004).

Most Scaphyglottis species grow epiphytically but sometimes they are found on broken branches as semiterrestrials. The most common habitats of those plants are humid forest, wet forest and cloud forest (Dressler 2001).

The geographical range of the genus extends from Mexico southward to Brazil and Bolivia. About $70 \%$ of the species were found in Costa Rica and Panama (Dressler 2001). Ortiz and Uribe (2007) placed 32 Scaphyglottis taxa on the list of Colombian orchids, including two species they classified as Reichenbachanthus. All of them except $S$. aurea (Rchb. f.) Foldats were found in lowlands and lower montane regions up to $2000 \mathrm{~m}$ a.s.l. (Ortiz Valdivieso \& Uribe Vélez 2007).

Our recent studies on Scaphyglottis in several Colombian herbaria revealed three distinctive species described here as new. Each is described, with taxonomic notes and information about its habitat, and illustrated by a line-drawing. The distribution of the new species is shown on a schematic map. 


\section{DESCRIPTIONS OF THE NEW SPECIES}

Scaphyglottis caquetana Szlach. \& Kolan., sp. nov.

Fig. 1

This species is characterized by having an elongate, 3-4-flowered inflorescence and almost quadrilobate lip with 3 prominent verrucose ridges running from the base of the lip almost to the apex.

Holotype: COLOMBIA, Caquetá. Mpio. Solano. Rio Mesay, Chorro Jacameya, extreme S de la Serranía de Chiribiquete, Alt. 200-300 m, 25 Nov. 1995, D. Cárdenas, N. Rodriguez, J. Manaidego \& M. Yukuna 6899 (COAH).

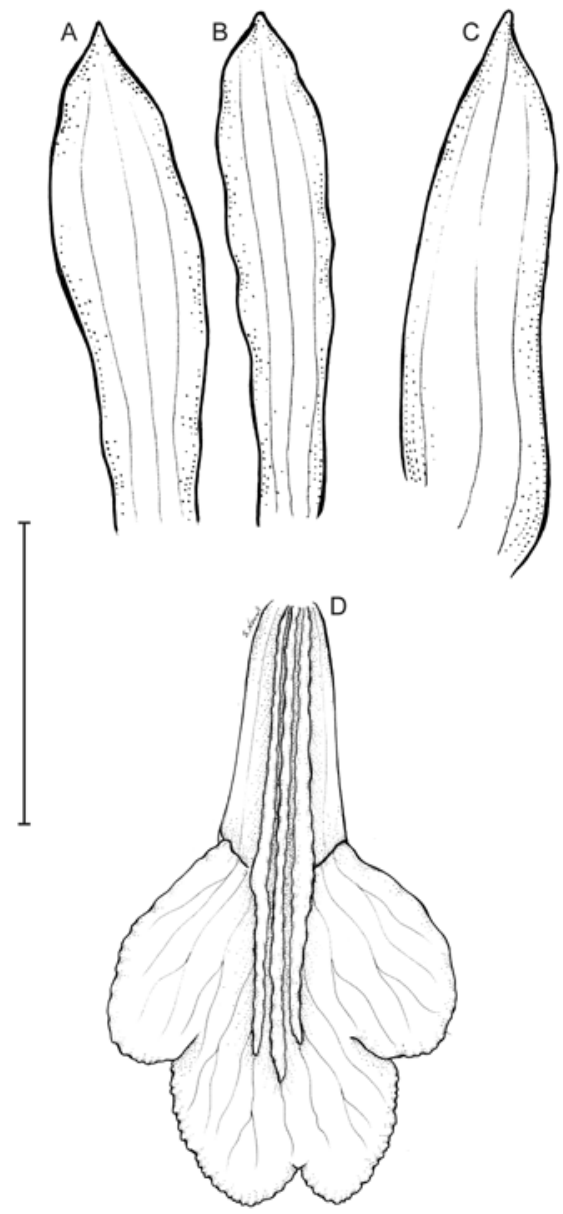

Fig. 1. Scaphyglottis caquetana Szlach. \& Kolan., sp. nov. A - dorsal sepal; B - petal; C - lateral sepal; D - lip. Scale bar $=4 \mathrm{~mm}$. Drawn by S. Nowak from holotype.
Pseudobulbs to $13 \mathrm{~cm}$ long and $3 \mathrm{~mm}$ in diameter, fusiform, bifoliate. Leaves up to $11 \mathrm{~cm}$ long and $3 \mathrm{~mm}$ wide, linear-lanceolate, acute. Inflorescence to $1.5 \mathrm{~cm}$ long, laxly 3-4-flowered. Flowers medium-sized. Floral bracts $c a 2 \mathrm{~mm}$ long. Pedicel and ovary $9 \mathrm{~mm}$ long. Dorsal sepal $7.5 \mathrm{~mm}$ long, $1.8 \mathrm{~mm}$ wide, oblong-oblanceolate, acute to acuminate, 3-nerved. Petals $7.5 \mathrm{~mm}$ long and $1.5 \mathrm{~mm}$ wide, linear-ligulate, acute to subobtuse, subfalcate, 3-nerved. Lateral sepals $8 \mathrm{~mm}$ long, $2 \mathrm{~mm}$ wide, ligulate, acuminate, subfalcate, 3-nerved. Lip $8 \mathrm{~mm}$ long in total, $4 \mathrm{~mm}$ wide when spread, clawed, with 3 verrucose ridges running from base almost to apex; claw 3.0-3.5 mm long; lamina up to $5 \mathrm{~mm}$ long, quadrilobate in middle, lobes elliptic-ovate, rounded, somewhat crispate along margins. Gynostemium $5 \mathrm{~mm}$ long.

ETymology. In reference to the department where the type specimen was collected.

Distribution AND ECOlOGY. Known so far from the Colombian department of Caquetá. Alt. 200-300 m. Flowering in November.

Notes. Unique in the genus for its peculiar, almost quadrilobate lip with 3 verrucose ridges almost the entire length.

Scaphyglottis obtusisepala Szlach. \& Kolan., sp. nov.

Fig. 2

Species somewhat similar to Scaphyglottis stellata Lodd. ex Lindl. but lip callus very broad, spread between lip lateral lobes, sepals and petals obtuse, petals widest at apex, sepals ligulate-elliptic. Also similar to $S$. modesta (Rchb. f.) Schltr. from which it differs by having bifoliate pseudobulbs and different lip callus.

Holotype: COLOMBIA, Caquetá. Mpio. Belen de los Andaquies. Cordillera Oriental, vertiente oriental. Parque-Bosque Microcuenca la Resaca, $1^{\circ} 26^{\prime} 23.5^{\prime \prime} \mathrm{N} 75^{\circ} 53^{\prime} 24.5^{\prime \prime} \mathrm{W}$, Alt. $720 \mathrm{~m}, 10$ Oct. 2007, W. Trujillo, J.A. Rios, H. Gonzales \& R. Alope WT1042 (COAH).

Pseudobulbs to $10 \mathrm{~cm}$ long and $5 \mathrm{~mm}$ in diameter, fusiform, bifoliate. Leaves up to $12 \mathrm{~cm}$ long and $4 \mathrm{~mm}$ wide, linear, acute. Inflorescence 


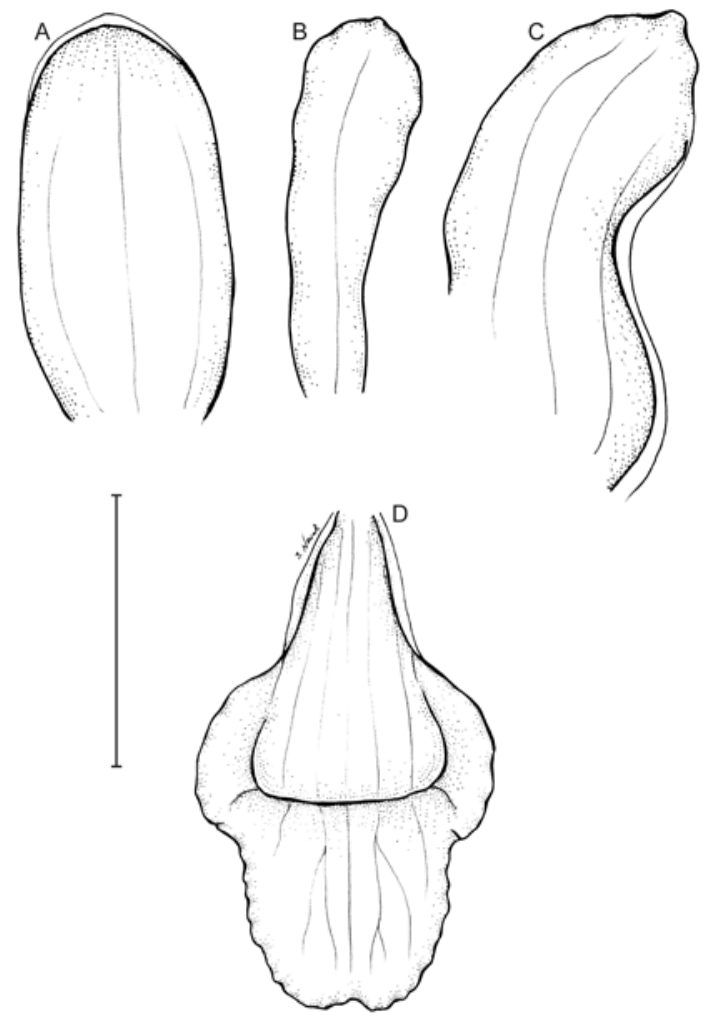

Fig. 2. Scaphyglottis obtusisepala Szlach. \& Kolan., sp. nov. A - dorsal sepal; B - petal; C - lateral sepal; D - lip. Scale bar $=2 \mathrm{~mm}$. Drawn by S. Nowak from holotype.

fasciculate, several-flowered. Flowers small, sepals somewhat fleshy. Pedicel and ovary $4 \mathrm{~mm}$ long. Dorsal sepal $3 \mathrm{~mm}$ long, $1 \mathrm{~mm}$ wide, elliptic to oblong-elliptic, obtuse, concave, obscurely 3-nerved. Petals $3 \mathrm{~mm}$ long, $0.8 \mathrm{~mm}$ wide, linear, somewhat widened towards apex, rounded, subfalcate, 1-nerved. Lateral sepals $3.5 \mathrm{~mm}$ long, $1 \mathrm{~mm}$ wide, ligulate-elliptic, falcate, obtuse, concave at base, obscurely 3-nerved. Lip $3.5 \mathrm{~mm}$ long in total and $1.5 \mathrm{~mm}$ wide when expanded; claw channelled; lamina 3-lobed near middle; middle lobe elliptic-subquadrate, truncate or emarginate at apex, somewhat erose on margins; lateral lobes obliquely obovate; callus prominent, spread between lateral lobes. Gynostemium $2.3 \mathrm{~mm}$ long.

ETymology. An allusion to the presence of obtuse sepals.
Distribution AND ECOLOGY. Found so far on the eastern slopes of Eastern Cordillera in the Colombian department of Caquetá. Alt. $720 \mathrm{~m}$. Flowering in October.

Notes. The most distinguishing character of this species consists in its fleshy sepals, which are obtuse at the apex. The lip callus is very prominent and spread between the lip lateral lobes.

\section{Key to the COLOMBIAN SPECIES OF SCAPHYGLOTTIS}

1. Lip immovable ................... 2

1. Lip movable ...................... 3

2. Lateral sepals linear-lanceolate, lip lateral lobes rounded .......S. emarginata (Garay) Dressler

2. Lateral sepals obliquely oblong ovate to ovatelanceolate, lip lateral lobes obtuse-triangular ... S. reflexa Lindl.

3. Lip distinctly 3 -lobed $\ldots \ldots \ldots \ldots \ldots \ldots \ldots 4$

3. Lip entire or obscurely 3 -lobed. . . . . . . . . 20

4. Inflorescence consisting of 1-2 flowers ..... 5

4. Inflorescence consisting of more than two flowers

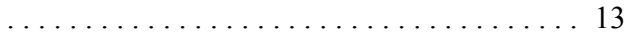

5. Leaves grass-like, usually less than $5 \mathrm{~mm}$ wide. . 6

5 . Leaves linear-lanceolate to elliptic, usually more than $5 \mathrm{~mm}$ wide ................... 8

6. Lip middle lobe subquadrate ... S. exilis Schltr.

6. Lip middle lobe subtriangular . . . . . . . . . 7

7. Lip middle lobe acuminate, lateral lobes rounded ..................... chocoana I. Bock

7. Lip middle lobe acute, lateral lobes obtuse ...... . S. longicaulis S. Watson

8. Leaves linear-ligulate, bilobulate at apex ...9 8. Leaves lanceolate, acute . . . . . . . . . . . . . . 11

9. Lip middle lobe decurved, subquadrate, margins crenulate . . . . . . . . S. triloba B. R. Adams

9. Lip middle lobe not decurved, suborbicular-flabellate or subquadrate, margins not crenulate . . . . . 10

10. Lip middle lobe widest at base, rounded at apex ........ S. bilineata (Rchb. f.) Schltr. $10^{*}$ Lip middle lobe widest at apex, truncate ..... S. lueckelii I. Bock

11. Lateral lobes obliquely subquadrate

S. stricta Schltr.

$11^{*}$ Lateral lobes obliquely obovate ......... 12

12. Lip middle lobe rounded, obtuse. ..........

S. boliviensis (Rolfe) B. R. Adams 
12. Lip middle lobe long acuminate, acute......

S. huebneri Schltr.

13. Middle lobe of lip emarginate, retuse ...... 14 13. Middle lobe of lip apiculate, acute to obtuse .....

S. modesta (Rchb. f.) Schltr.

14. Lip longer than tepals . . . . . . . . . . . 15

14 . Lip subequal in length to tepals. . . . . . . 16

15. Leaves elliptic to oblong-lanceolate ........... ........... S. bifida (Rchb. f.) C. Schweinf.

$15^{*}$. Leaves linear-oblong

S. behrii (Rchb. f.) Benth. \& Hook. f. ex Hemsl.

16. Lip middle lobe deeply bilobulate ......... ........... S. caquetana Szlach. \& Kolan.

16. Lip middle lobe undivided. . . . . . . . . 17

17. Leaf $c a 1 \mathrm{~mm}$ wide ....................

S. filifolius Szlach. \& Kolan.

$17^{*}$. Leaf more than $3 \mathrm{~mm}$ wide. . . . . . . . . 18

18. Lip callus very broad, spread between lateral lobes and not exceeding them .

S. obtusisepala Szlach. \& Kolan. 18*. Lip callus narrow, exceeding lateral lobes ....

19. Lip 3.0-3.5 mm long, middle lobe elliptic-obovate S. violacea Lindl.

19. Lip 6.0-8.5 $\mathrm{mm}$ long, middle lobe ligulate to rectangular. . . . . . . . . S. stellata Lodd. ex Lindl.

20. Inflorescence a fascicle of more than 2 flowers

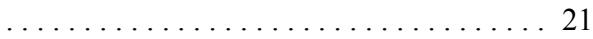

$20 *$ Inflorescence $1-2$-flowered . . . . . . . . 22

21. Lip $c a 10 \mathrm{~mm}$ long and $5 \mathrm{~mm}$ wide, subpandurate

S. coriacea (L. O. Williams) Dressler

21. Lip 2.5-4.0 $\mathrm{mm}$ long and 1.5-2.5 mm wide, obovate to oblong . . . . S. S. minutiflora Ames \& Correll

22. Leaves oblong-elliptic to elliptic-lanceolate...

......................... 23

22 . Leaves linear . . . . . . . . . . . . 25

23. Pedicellate ovary $c a 20 \mathrm{~mm}$ long, lip panduriformobovate. ..... S. arctata (Dressler) B. R. Adams

23. Pedicellate ovary $c a 3-4 \mathrm{~mm}$ long, lip spathulate ....................... 24

24. Sepals up to $3.5 \mathrm{~mm}$ long, petals subobtuse .. ..................... sickii Pabst 24. Sepals up to $2.5 \mathrm{~mm}$ long, petals emarginate. .

S. signata I. Bock

25. Sepals linear-lanceolate, ligulate to lanceolate....

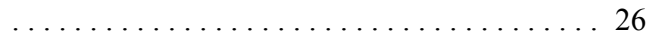

25 . Sepals elliptic-ovate. . . . . . . . . . . 28

26. Lip spathulate to cuneate-flabellate. . . . . 27 26. Lip oblong-ovate .

S. isochiloides (Regel) Foldats
27. Lip middle lobe conspicuous

S. esuriens (Rchb. f.) Schltr.

27. Lip middle lobe inconspicuous . . . . . . . . . . .

S. fusiformis (Griseb.) R.E. Schultes

28. Lip anchoriform

S. gentryi Dodson \& M. Monsalve

28. Lip subquadrate, cuneate-ovate, oblong-lanceo-

late to ovate . . . . . . . . . . . . . . . 29

29. All tepals similar in size and shape. . . . . . 30

29. Petals and sepals dissimilar........... 33

30. Leaves less than $5 \mathrm{~mm}$ wide............. $\ldots \ldots \ldots \ldots$ S. sessilis (Rchb. f.) Foldats

30. Leaves more than $5 \mathrm{~mm}$ wide . . . . . . . 31

31. Tepals elliptic to ovate-elliptic ............

S. aurea (Rchb. f.) Foldats

31* Tepals lanceolate-oblong to ovate-oblong. . . . 32

32. Stems usually simple, or with only few

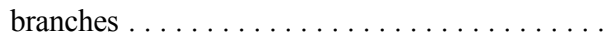

...... S. punctulata (Rchb. f.) C. Schweinf.

32. Stems branched . . . . . . . . . . . . .

$\ldots \ldots \ldots \ldots$ S. summersii L. O. Williams

33. Lip flabellate, cuneate-ovate . . . . . . . . . 34 33. Lip subquadrate to ovate-quadrate

S. livida (Lindl.) Schltr.

34. Pseudobulbs stipitate . . . . . . . . . . . . . .

S. graminifolia (Ruiz \& Pav.) Poepp. \& Endl.

34. Pseudobulbs cylindric-fusiform. . . . . . . . . .

S. prolifera (Sw.) Cogn.

ACKNOWLEDGEMENTS. We thank the curators and staff of the cited herbaria for their kind hospitality and assistance during visits and for making specimens available on loan, the anonymous reviewers for helpful remarks on the manuscript, and Sławomir Nowak for preparing the illustrations. The research described here was funded by the Polish Ministry of Science and Higher Education (grant no. 8124/B/PO1/2011/40).

\section{REFERENCES}

ADAms B. R. 1988. New species and combinations in the genus Scaphyglottis. Phytologia 64: 249-258.

Ames O., Hubbard F. T. \& Schweinfurth Ch. 1934. Critical notes on Costa Rican orchids. Bot. Mus. Leafl. 3: 37-42.

Dressler R. L. 1994. Proposal to conserve Scaphyglottis against Hexisea (Orchidaceae). Taxon 43: 665-666.

Dressler R. L. 2001. Scaphyglottis. In: A. M. Pridgeon, P. J. Cribb, M. W. Chase \& F. N. Rasmussen (eds), Genera Orchidacearum: 4: Epidendroideae (Part 1), pp. 310-313. Oxford University Press, Oxford. 
DRESSLER R. L. 2002. New species and combinations in Costa Rican orchids II. Lankesteriana 3: 25-29.

DRESSLER R. L. 2004. Der Scaphyglottis pulchella Komplex; The Scaphyglottis pulchella complex. J. Orchideenfreund 11: 305-315.

Dressler R. L., Whitten M. \& Williams N. H. 2004. Phylogenetic relationships of Scaphyglottis and related genera
(Laeliinae: Orchidaceae) based on nrDNA ITS sequence data. Brittonia 56: 58-66.

Ortiz VÁldivieso P. \& Uribe Vélez C. 2007. Galería de Orquideas de Colombia (CD edition). Asociación Bogotana de Orquideología, Bogotá.

Poeprig E. F. \& Endlicher S. L. 1836. Nova Genera et Species Plantarum 1(7-10). Sumptibus F. Hofmeister, Lipsiae.

Received 8 October 2014 